\title{
Prevalence of Malaria in Catchment Area of Khairpure Medical College Hospital Khairpure Mirs Pakistan
}

\author{
Badvi JA1*, Jawed $\mathrm{M}^{2}$ and Siddiqui $\mathrm{SA}^{3}$ \\ 1Professor and head of Pathology Department, Khairpure Medical College, Pakistan \\ ${ }^{2}$ Post graduate Researcher, Liaquat University of health sciences, Pakistan \\ ${ }^{3}$ Senior Pathologist, Khairpure Medical College Hospital, Pakistan
}

\section{Research Article \\ Volume 2 Issue 2}

Received Date: October 03, 2017

Published Date: October 28, 2017

*Corresponding author: Jawed Ahmed Badvi, Professor and head of Pathology Department, Khairpure Medical College, Khairpure Mirs, Pakistan, E-mail: jawedbadvi1958@gmail.com

\section{Abstract}

Plasmodium vivax and Plasmodium falciparum are prevalent in Khairpure Mirus, Pakistan, yet up-to-date data on the epidemiology of malaria in Khairpure Mir's catchment area, Pakistan are not available. This study was undertaken to determine the current prevalence and distribution of Plasmodium species first time in Khairpure medical college Khairpure Mirus catchment area.

\section{Objectives}

To identify the common species in KHAIRPURE VICINITY.

Trend of specie first insight study malarial parasites In Khairpure medical college hospital catchment area.

To take the effective measures to control the malarial cases as it causes the serious illness, cerebral malaria and black water fever.

Material and Methods: A is data collected from Khairpure Medical college laboratory from January 2017 to august 2017 to assess the prevalence, trend of malariositemia, and species. Population survey was conducted in 2017 using blood samples collected febrile patients of all ages from OPD of Khairpure medical college Khairpure catchment area. Microscopically Blood sample was taken maked thin and thick film according to protocol confirmed microscopically, Plasmodium-positive blood samples were reconfirmed by chromatography.

Conclusion: Plasmodium infections in Pakistan are largely attributed to P. vivax but P. falciparum and mixed species infections are also prevalent in Khairpure medical college hospital catchment area. In addition, regional variation in the prevalence and species composition of malaria is high.

Keywords: Prevalence; Plasmodium falciparum; Plasmodium vivax; Malaria; Khairpure Mir's; Pakistan 


\section{Vaccines \& Vaccination Open Access}

\section{Introduction}

The World Health Organization (WHO) estimates the burden of malaria to be 198 million cases with 584,000 deaths in the year 2013 being a major cause of poverty and low productivity and vice versa [1]. Despite the fact that decreasing numbers of malaria cases and mortality are recently reported in some endemic regions, most countries in sub-Saharan Africa still suffer from an immense burden of malaria mortality. Data indicates that $90 \%$ of global deaths due to malaria occur in Africa, especially among children under age five who are the hardest hit with at least $75 \%$ of the fatalities [1].

During the time when the study described here was performed, malaria accounted in Ghana for 32\% of all outpatient department (OPD) visits and 49\% hospital admissions in children under 5 years of age and morbidity was about three million cases with 4,000 deaths annually [2]. Infants are thought to be protected against malaria during the first 6 months of life, largely due to the transfer of maternal antibodies and the presence of fetal hemoglobin globally; malaria causes around 627,000 deaths in 2013, mostly of children aged less than 5 years living in Africa [1,3,4]. There are now a large number of regular prevalence surveys of childhood parasitaemia, as most malaria deaths occur in children, but published surveys of parasitaemia in the general adult population are very scarce $[1,4,5]$. Thus, of the 46 countries contributing household surveys of the presence of malaria parasites to the WHO 2013 report on malaria, all were of children [4]. The Kenya malaria survey 2007 found a prevalence of $17 \%$ in children under 5 years in endemic areas, compared with $1.4 \%$ in areas of seasonal malaria transmission, $1 \%$ in epidemic prone areas, and $0.4 \%$ in low risk transmission areas (Kenya malaria indicator survey 2007) [6]. The Kenya malaria indicator survey, 2010 , covered children up to the age of 14 and found that the prevalence in children below 5 years increased from $4 \%$ in 2007 to $8 \%$ in 2010 while the prevalence in children aged $10-15$ was $15 \%$ on average, ranging from $38 \%$ in the Lake endemic area to less than 5\% elsewhere [7].

Globally, malaria causes around 627,000 deaths in 2013, mostly of children aged under 5 years living in Africa [4]. There are now a large number of regular prevalence surveys of childhood parasitaemia, as most malaria deaths occur in children, but published surveys of parasitaemia in the general adult population are very scarce of the presence of malaria parasites in children aged 2-10 years between 1975 and 2009 indicates that malaria is declining in prevalence across the country over the last 20 years, but endemic areas remain around Lake Victoria basin and in the Coast region [4-6].

Ninety-five million of Pakistan's 161 million people, roughly $60 \%$ of Pakistan's population, live in malariaendemic regions $[8,9]$. After eradication efforts in the 1960 s, malaria surged back to an epidemic level in the 1970s. In recent years, an uptick in malaria can be partially attributed to floods that affected approximately 20 million people in over 60 districts [9]. Despite a wellestablished malaria control programme, 500,000 malaria infections and 50,000 malaria-attributable deaths occur each year in Pakistan, with approximately $37 \%$ of cases estimated to occur in regions along the borders with Afghanistan and Iran [10,11].

Pakistan's climate ranges from tropical to temperate with dry conditions along the southern coast, and elevation ranges from sea level to nearly 9,000 meters $[12,13]$. Plasmodium vivax (responsible for approximately $64 \%$ of infections) and Plasmodium falciparum (causing $36 \%$ of infections) are the two prevalent Plasmodium species in Pakistan, and malaria is primarily found in the provinces of Khyber Pakhtunkhwa, Balochistan, Sindh and the Federally Administered Tribal Areas [11,14]. Malaria transmission is considered to be unstable, with major $P$. vivaxtransmission peaking from June to September and again in April to June, when relapses of infections acquired the previous season are observed [15]. The major transmission period for P. falciparum in Pakistan is between August and December [15].

It has been estimated that in countries where P. vivax is predominant, over one-third of patients presenting with acute $P$. vivax also harbour cryptic $P$. falciparum [16].

The rise of $P$. falciparum in parts of Pakistan may be partially attributable to failed treatment of chloroquineresistant infections [2]. Chloroquine resistance in $P$. falciparum was reported for the first time from Pakistan in 1984 and later confirmed as widespread [17-20]. A recent study found that $90 \%$ of $P$. falciparum samples collected in the provinces of Balochistan and Sindh carried the pfcrt $76 \mathrm{~T}$ allele responsible for conferring chloroquine resistance [21]. Although chloroquine is recommended only for treatment of $P$. vivax, $P$. falciparum infections are often treated with chloroquine, as presumptive diagnosis or empiric treatment is usually based on clinical symptoms in resource-limited countries like Pakistan [22]. Two contributors to the low level of malaria in Punjab have been suggested. Environmental changes resulting in water logging and salinization may 


\section{Vaccines \& Vaccination Open Access}

have reduced suitable habitats for the more efficient vector, Anopheles culicifacies, while increasing breeding sites for the salt tolerant and less efficient vector, Anopheles stephensi [11]. Additionally, overall socio-economic conditions and the public health infrastructure are comparatively better in Punjab than in the rest of the country, and may play a role in more effectively controlling malaria [23].

The heavy influx and continued presence of refugees from Afghanistan, where P. falciparum is more prevalent, may also contribute to the increase of $P$. falciparum in Pakistan [24-26] potentiating clinical trials of falciparum malaria in the refugee populations in regions such as Khyber Pakhtunkhwa province [27]. Studies have indicated that refugees have low immunity and are more susceptible to malaria in Pakistan than are permanent residents [28-30]. These factors may lead to increased disease and transmission in the refugee population. Monitoring the distribution and burden of Plasmodium species in Pakistan as changes occur in both human and parasite populations is necessary to ensure appropriate treatment, particularly in situations where diagnosis by microscopy or species-specific rapid diagnostic tests is not available.

A handful of malaria epidemiological studies have been conducted in parts of Pakistan, but the assessment of the malaria burden on a national scale has been hindered by the lack of data from many areas [31]. One report of malariometric surveys conducted from 2005 to 2009 found both spatial and temporal variation in malaria endemicity. Malaria prevalence was highest in the Federally Administered Tribal Areas, followed by Baluchistan and Khyber Pakhtunkhwa Provinces [27], and over the five-year period malaria incidence was highest in 2009. The aim of this study was to provide current report of malaria in Khairpure medical college hospital catchment area Khairpure mirus Pakistan. Surveys were conducted o OPD basis from January 2017 to August 2017 to determine the prevalence of malaria infection among suspected cases and to map the distribution of Plasmodium species across the in Khairpure medical college hospital catchment area Khairpure Mirus Pakistan.

The two sites in Sindh, Karachi and Hyderabad, had an overall malaria prevalence of $11 \%$, with very few $P$. falciparum or mixed species infections. In contrast, previous studies have shown that approximately onequarter to one-half of malaria cases were attributed to $P$. falciparum in these cities [32-34]. This difference may be attributable to the narrow sampling window in this province. The majority of samples were collected in April in Sindh, while previous observations indicate that peak infection of $P$. falciparum does not occur until later in the year [35].

\section{Material and Methods}

A is data collected from Khairpure Medical college laboratory from January 2017 to august 2017 to assess the prevalence, trend of malariositemia, and species. Population survey was conducted in 2017 using blood samples collected febrile patients of all ages from OPD of Khairpure medical college Khairpure catchment area. Microscopically Blood sample was taken make thin and thick film according to protocol confirmed microscopically, Plasmodium-positive blood samples were reconfirmed by chromatography.

\section{Results}

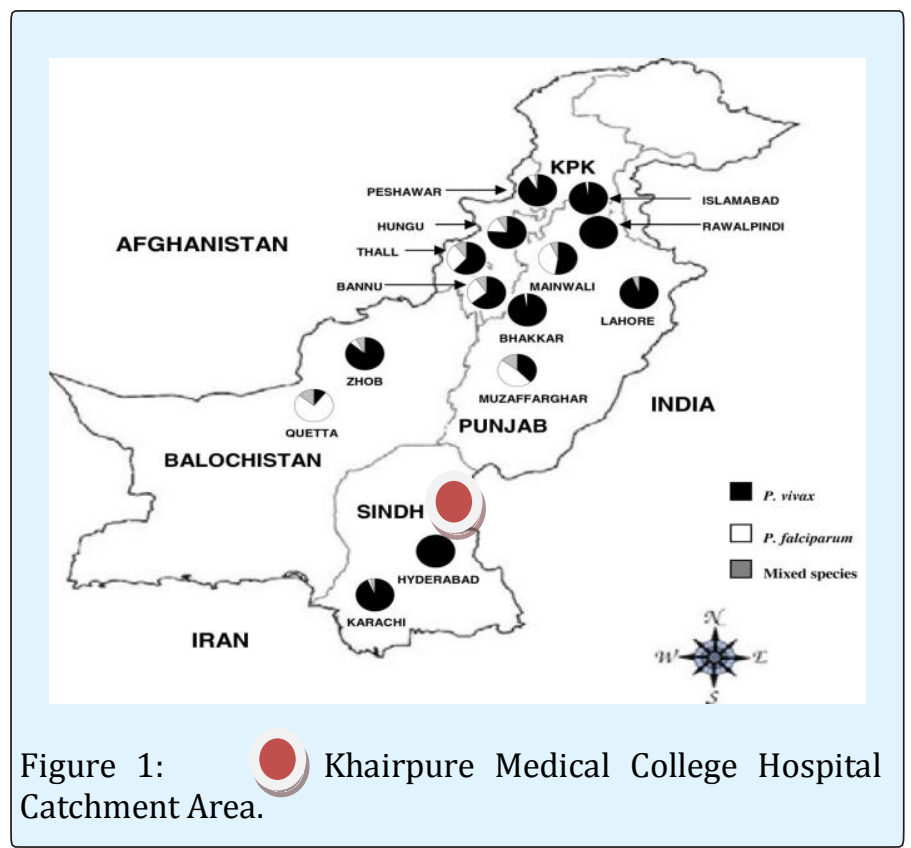

This study was conducted on OPD cases attended hospital from January 2017 To August 20017A total of 2001, Blood sample was collected from the entire patient thick and thin film was prepared and identified microscopically. By microscopy, $1.5 \% \quad(\mathrm{n}=31)$ were identified as P. falciparum, $2 \%(\mathrm{n}=415)$ as $P$. vivax .of the 20017,314 and female were 98 and children under 14 years of age were 33.The highest rate of positive cases were in August 2017 may be due to rainy area and flood located area rise of ware in river and canals along with 


\section{Vaccines \& Vaccination Open Access}

hot season in that area and highest cases were in Male due to labouruos work in their land in the sun set time and no awareness about the warring of cloths properly, of the 20017 cases $446,2.2 \%$ (Figure 1 \& Table 1 ).

\section{Discussion}

This survey was conducted first insight study in the Khairpure Medical college catchment area. To provide upto date information on malaria prevalence throughout the Khairpure Medical college catchment area in Pakistan. Because samples were collected from outdoor patients presenting with symptoms, one limitation is the potential for regional variation in treatment-seeking behavior and access to treatment centers. AS from result of the 20017 visited cases only, 446, $2.2 \%$ percent is very negligible number of cases, and can say Malaria is under controller in this area able figure, which create the hope of eradication, needs the effective measures of prevention, which may be net during sleep, netting in rooms and the houses, Proper wearing of clothes, Clear the ponds in the villages, insecticide spry in routine, in this way we will prevent the Dengue also. This of type data is needed yearly should be should be evaluated yearly, by keeping this study as a base line study for comparing weather malaria is controlled, rising or eradicating trend (Figures $2 \& 3)$.

\begin{tabular}{|c|c|c|c|c|c|c|c|c|}
\hline Month & Total Malaria Cases & Vivax & Falciparum & Male & Female & Child & $\begin{array}{c}\text { Age in } \\
\text { years }\end{array}$ & $\begin{array}{c}\text { Total } \\
\text { Positive }\end{array}$ \\
\hline January & 2007 & 30 & 4 & 19 & 11 & 4 & $5-14$ & 34 \\
\hline February & 2045 & 33 & 2 & 22 & 10 & 3 & $5-14$ & 35 \\
\hline March & 2661 & 38 & 4 & 32 & 8 & 2 & $5-14$ & 42 \\
\hline April & 2421 & 23 & 2 & 16 & 5 & 4 & $5-14$ & 25 \\
\hline May & 2683 & 60 & 2 & 45 & 9 & 8 & $5-14$ & 62 \\
\hline June & 2164 & 56 & 5 & 42 & 15 & 3 & $5-14$ & 61 \\
\hline July & 2708 & 80 & 5 & 60 & 20 & 5 & $5-14$ & 85 \\
\hline August & 3351 & 95 & 7 & 78 & 20 & 4 & $5-14$ & 102 \\
\hline
\end{tabular}

Table 1: Prevalence of Malaria in Catchment Area of Khairpure Medical College Hospital Khairpure Mirs Pakistan from January 2017 to August 2017.

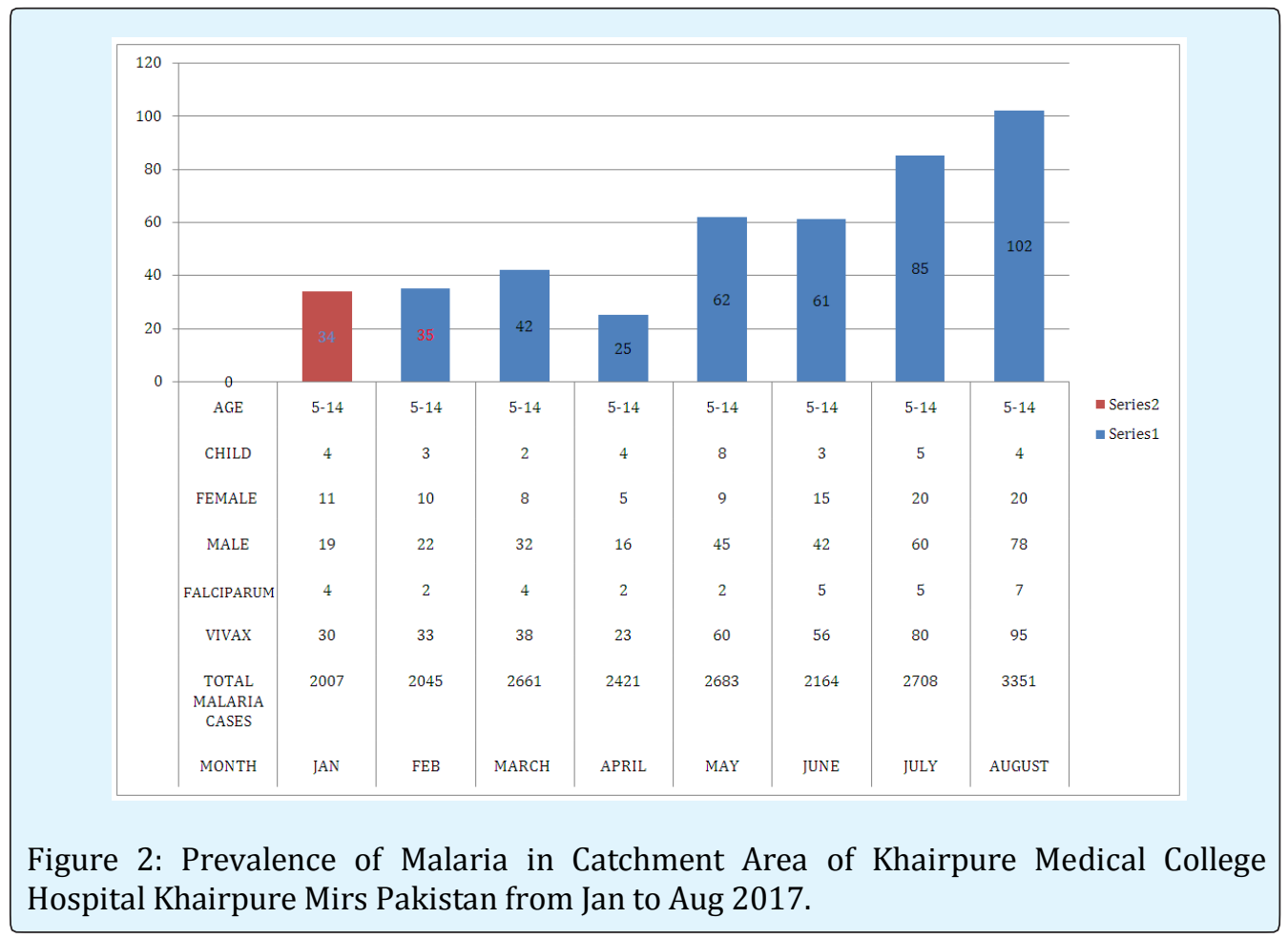

Badvi JA, et al. Prevalence of Malaria in Catchment Area of Khairpure Medical College Hospital Khairpure Mirs Pakistan. Vaccines Vaccin 2017, 


\section{Vaccines \& Vaccination Open Access}

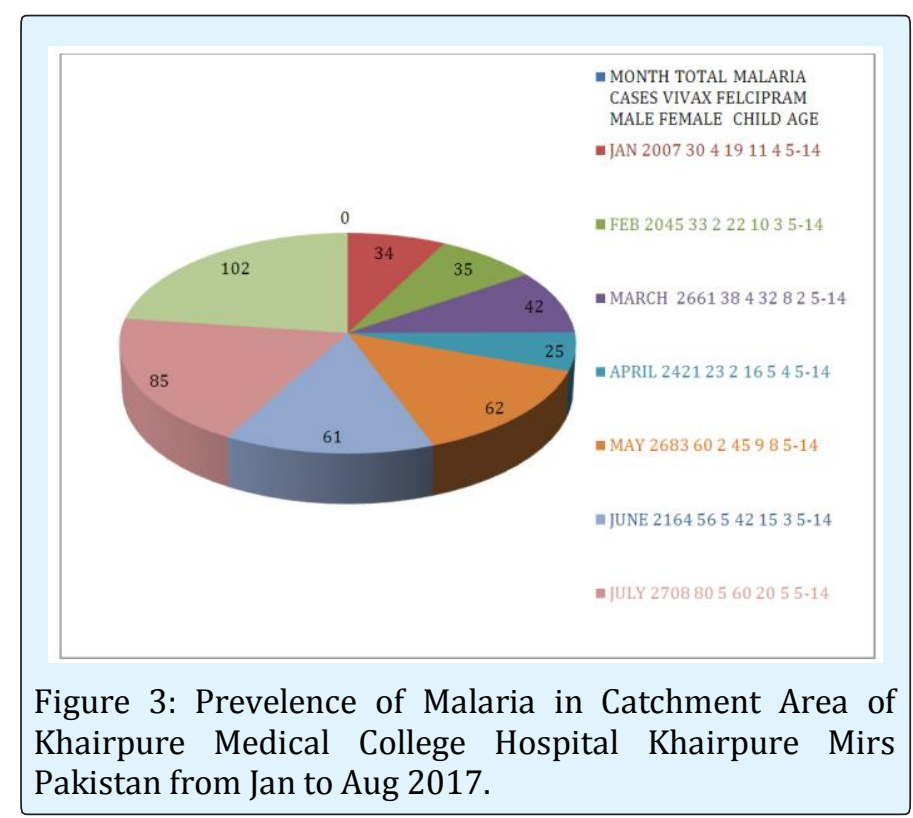

\section{References}

1. WHO (2014) World Malaria Report 2014. World Health Organization, Geneva.

2. WHO (2011) World Malaria Report 2011. World Health Organization, Geneva.

3. Pasvol G, Weatherall DJ, Wilson RJ (1977) Effects of foetal haemoglobin on susceptibility of red cells to Plasmodium falciparum. Nature 270: 171-173.

4. WHO (2013) The world malaria report. WHO, Geneva.

5. Mayor A, Aponte JJ, Saute F, Greenwood B, Dgedge M, et al. (2007) The epidemiology or malaria in adults in a rural area of southern Mozambique. Malar J 6: 3.

6. Awes AA, Buluma RCB, Bore J, Waweru P, Kundu F, et al. (2007) Kenya Malaria Indicator Survey. Kenya National Bureau of Statistics.

7. (2010) Kenya Malaria Indicator Survey. Division of Malaria Control, Ministry of Public Health and Sanitation. Kenya National Bureau of Statistics.

8. WHO (2013) WHO Eastern Mediterranean regional office. Cairo: World Health Organization.

9. Williams 0, Meek S (2011) Malaria: country profiles. London: Department of International Development.

10. Mukhtar M (2006) Killer number one: the fight against malaria: malaria strategy lags behind the global goals, Humanitarian news and analysis a service of the UN Office for the Coordination of Humanitarian Affairs. Nairobi: IRIN.

11. Kakar Q Khan MA, Bile KM (2010) Malaria control in Pakistan: new tools at hand but challenging epidemiological realities. East Mediterr Health J 16: S54-S60.

12. (2012) Federal Research Division: Country profile: Pakistan. Library of Congress.

13. Land surface elevation extremes by country.

14. WHO (2012) WHO: World malaria report 2012. World Health Organization, Geneva.

15. Bouma MJ, Dye C, van der Kaay HJ (1996) Falciparum malaria and climate change in the northwest frontier province of Pakistan. Am J Trop Med Hyg 55(2): 131-137.

16. Looareesuwan S, White NJ, Chittamas S, Bunnag D, Harinasuta $\mathrm{T}$ (1987) High rate of Plasmodium vivax relapse following treatment of falciparum malaria in Thailand. Lancet 2(8567): 1052-1055.

17. Nizamani MA, Kalar NA, Khushk IA (2006) Burden of malaria in Sindh, Pakistan: a two years surveillance report. J Liaquat University of Med Health Sci 5: 762783.

18. Ghanchi NK, Ursing J, Beg MA, Veiga MI, Jafri S, et al. (2011) Prevalence of resistance associated polymorphisms in Plasmodium falciparum field isolates from southern Pakistan. Malar J 10: 18.

19. Rana MS, Tanveer A (2004) Chloroquine resistance and Plasmodium falciparum in Punjab, Pakistan during 2000-2001. Southeast Asian J Trop Med Public Health 35(2): 288-291.

20. Robinson DS, Hadley-Brown M, Ejele OA, Robinson PS (1984) Chloroquine-resistant malaria in Pakistan. Lancet 2: 987.

21. Rawasia WF, Sridaran S, Patel JC, Abdallah J, Ghanchi NK, et al. (2012) Genetic backgrounds of the Plasmodium falciparum chloroquine resistant transporter (pfcrt) alleles in Pakistan. Infect Genet Evol 12(2): 278-281.

22. Parikh R, Amole I, Tarpley M, Gbadero D, Davidson M, et al. (2010) Cost comparison of microscopy vs. empiric treatment for malaria in Southwestern Nigeria: a prospective study. Malar J 9: 371. 


\section{Vaccines \& Vaccination Open Access}

23. Herrel N, Amerasinghe FP, Ensink J, Mukhtar M, van der Hoek W, et al. (2004) Adult anopheline ecology and malaria transmission in irrigated areas of South Punjab, Pakistan. Med Vet Entomol 18(2): 141-152.

24. Malik IA, Iqbal I, Malik S (1996) A study of cerebral malaria at Multan. Pakistan Pediatric J 20(4): 175180.

25. Murtaza G, Memon IA, Noorani AK (2004) Malaria prevalence in Sindh. Med Channel 10(2): 41-42.

26. Sheikh AS, Sheikh AA, Sheikh NS, Paracha SM (2005) Endemicity of malaria in Quetta. Pakistan J Med Res 44(1): 41-45.

27. Howard N, Durrani N, Sanda S, Beshir K, Hallett R, et al. (2011) Clinical trial of extended-dose chloroquine for treatment of resistant falciparum malaria among Afghan refugees in Pakistan. Malar J 10: 171.

28. Bouma MJ, Parvez SD, Nesbit R, Winkler AM (1996) Malaria control using permethrin applied to tents of nomadic Afghan refugees in northern Pakistan. Bull World Health Organ 74(4): 413-421.

29. Suleman M (1988) Malaria in Afghan refugees in Pakistan. Trans R Soc Trop Med Hyg 82(1): 44-47.
30. De ZJ, Mujtaba SM, Shah IH (1980) Malaria control and long-term periodicity of the disease in Pakistan. Trans R Soc Trop Med Hyg 74(5): 624-632.

31. Khan HU, Khattak AM, Khan MH, Mahsud IU, Shah SH (2006) A study of prevalence of malaria in adult population of D. I. Khan, Pakistan. Biomedica 22: 99104.

32. Noor M, Akbar H (2003) Prevalence of malaria in general population of distric Buner. J Pak Inst Med Sci 17(1): 75-80.

33. Beg MA, Sani N, Mehraj V, Jafri W, Khan MA, et al. (2008) Comparative features and outcomes of malaria at a tertiary care hospital in Karachi, Pakistan. Int J Infect Dis 12(1): 37-42.

34. Hayyat AS, Memon F, Shaikh N, Fatah Dero AF (2009) Incidence and pattern of malarial infection at a tertiary care Hospital of Hyderabad. World J Med Sci 4(1): 9-12.

35. (2010) MOH: Pakistan national strategic plan for malaria control 2010-2015. Islamabad: Ministry of Health. 